\title{
INFLUENCE DU STOCKAGE ET DES TRAITEMENTS INDUSTRIELS SUR LA TENEUR EN VITAMINES DU LAIT DE VACHE
}

\author{
par \\ Bernard BLANC \\ Institut Suisse des Vitamines et \\ Département scientifique Union Laitière Vaudoise. \\ Lausanne (Suisse)
}

Le lait, liquide biologique, est apparenté au sang au double point de vue de la composition et de la signification physiologique. En effet, d'une part de nombreux constituants du sérum sanguin se retrouvent dans le lait, tandis que d'autres qui lui sont propres représentent l'équivalent de constituants sériques manquants. D'autre part, il y a similitude de fonctions de ces deux liquides maternels nourriciers, l'un, le sang destiné à transmettre au fœtus les éléments indispensables à sa maturation, l'autre, le lait, assurant le développement optimal du nouveau-né. Le lait peut être considéré comme le prolongement biologique du sang; c'est son homologue externe et un sérum de survie pour les mammifères.

Le lait apparaît donc comme un aliment précieux que dès l'aube de l'histoire l'homme s'est assuré en domestiquant des mammifères ruminants. Au cours des guerres, le maintien de rations suffisantes de lait pour les enfants et les femmes enceintes ou allaitant leur bébé a toujours été au premier plan des soucis de ceux auxquels il incombait de prendre des mesures pour le ravitaillement des populations. Aujourd'hui encore, les efforts faits par des organisations telles que la FAO pour favoriser l'expansion de l'industrie laitière dans les pays en voie de développement où la population est généralement sous-alimentée, prouve bien l'importance reconnue à cet aliment complet qu'est le lait.

Le tableau I indique le taux des diverses vitamines dans le lait de vache et le lait maternel, les besoins journaliers en vitamines de l'adulte et du nourrisson (Dietary Allowances U. S. A. 1964) et la quantité de lait de vache nécessaire pour satisfaire ces besoins.

A part la couverture en vitamines liposolubles qui est insuffisante, une consommation journalière normale de lait et de produits laitiers fournit, en plus des autres nutriments, la plus grande partie des vitamines hydrosolubles en quantités compatibles avec les besoins. 
TABLEAU I

Taux des vitamines dans les laits ( $L V=$ Lait de Vache, $L M=$ Lait Maternel) ; besoin journalier en vitamines et quantités de $L V$ quotidiennes nécessaires pour couvrir les besoins

\begin{tabular}{|c|c|c|c|c|c|c|}
\hline & \multicolumn{2}{|c|}{$\begin{array}{c}\text { Teneur moyenne } \\
\text { dans }\end{array}$} & \multicolumn{2}{|c|}{$\begin{array}{c}\text { Besoin journalier* } \\
\mathrm{mg} / \text { jour }\end{array}$} & \multicolumn{2}{|c|}{$\begin{array}{l}\text { LV nécessaire } \\
\text { pour couvrir le } \\
\text { besoin journalier } \\
1 \text { jour }\end{array}$} \\
\hline & $\begin{array}{l}\mathrm{LV} \\
\mathrm{mg} / \mathrm{l}\end{array}$ & $\begin{array}{l}\mathrm{LM} \\
\mathrm{mg} / \mathrm{l}\end{array}$ & Adulte & $\begin{array}{l}\text { Nour- } \\
\text { risson }\end{array}$ & Adulte & $\begin{array}{l}\text { Nour- } \\
\text { risson }\end{array}$ \\
\hline Liposolubles: & & & & & & \\
\hline 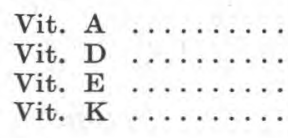 & $\begin{array}{l}0,27 \\
0,001 \\
0,6 \\
0,06\end{array}$ & $\begin{array}{l}0,61 \\
0,0012 \\
2,4 \\
0,015\end{array}$ & $\begin{array}{l}1,5 \\
0,01 \\
20 \\
4\end{array}$ & $\begin{array}{l}0,45 \\
0,01 \\
2 \\
1-2\end{array}$ & $\begin{array}{l}5,5 \\
10 \\
33,3 \\
66,7\end{array}$ & $\begin{array}{c}1,7 \\
10 \\
3,3 \\
33,3\end{array}$ \\
\hline Hydrosolubles : & & & & & & \\
\hline 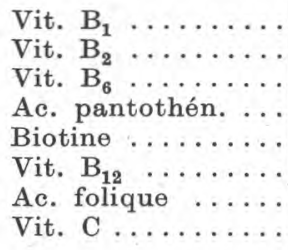 & $\begin{array}{l}0,43 \\
1,56 \\
0,70 \\
3,4 \\
0,057 \\
0,010 \\
0,0013 \\
11\end{array}$ & $\begin{array}{c}0,142 \\
0,373 \\
0,18 \\
2,46 \\
0,008 \\
\text { traces } \\
0,0014 \\
52\end{array}$ & $\begin{array}{l}1,0 \\
1,6 \\
1,5 \\
10 \\
0,225 \\
0,004 \\
0,1 \\
70\end{array}$ & $\begin{array}{l}0,4 \\
0,6 \\
0,4\end{array}$ & $\begin{array}{l}2,3 \\
1 \\
2,1 \\
2,9 \\
3,9 \\
0,4 \\
77 \\
6,4\end{array}$ & $\begin{array}{l}0,9 \\
0,4 \\
0,6\end{array}$ \\
\hline $\begin{array}{l}\text { Inositol } \ldots \ldots \ldots \\
\text { Ac. nicotine } \ldots \ldots\end{array}$ & $\begin{array}{l}80 \\
0,74\end{array}$ & $\begin{array}{l}449 \\
\quad 1,83\end{array}$ & $\begin{array}{r}1 \\
17\end{array}$ & 6 & $\begin{array}{l}0,01 \\
23\end{array}$ & 8,1 \\
\hline
\end{tabular}

* D'après Dietary allowances, USA, 1964.

Mais ce précieux aliment qu'est le lait est malheureusement une denrée extrêmement périssable et sa conservation est très limitée. C'est pourquoi, si l'on veut aujourd'hui en assurer une diffusion et une utilisation de plus en plus larges, on ne peut plus souscrire aux vues naturistes de certains adeptes de croyances prônant l'utilisation du lait tel que le fournit la Nature, sans aucune modification. Ce n'est au contraire qu'en augmentant sa conservabilité par des procédés industriels toujours mieux adaptés que l'on parviendra à une diffusion plus large du lait pour lui permettre 
ainsi de mieux remplir son rôle d'aliment essentiel. L'effet des divers traitements industriels est très variable d'une vitamine à l'autre. Nous passerons en revue les connaissances acquises au sujet des vitamines les mieux étudiées.

\section{Vitamine A et carotène}

En 1920 déjà, Hopkins montre l'influence de la chaleur en présence d'air sur la vitamine A ; par chauffage du beurre 105 minutes à $88^{\circ} \mathrm{C}$ ou stérilisation 50 minutes à $120^{\circ} \mathrm{C}$ il $\mathrm{y}$ a perte totale de l'activité vitaminique A. En l'absence d'air cependant, le beurre conserve une grande partie de ses vitamines $\mathrm{A}$, même après traitement de 4 heures à $120^{\circ} \mathrm{C}$.

Alors que certains (Drummond et Coward (1920), Zilva (1922), $E d d y$ et Kohmann (1925-1931) confirment la thermostabilité de la vitamine $\mathrm{A}$ en l'absence d'oxygène, d'autres auteurs précisent cette notion en mettant en évidence :

1) soit l'importance de la lumière, (Peacock 1926), Drummond et Hilditch (1930),

2) soit celle de l'oxydation (Holmes et coll. (1936), qui sont capables de catalyser à elles seules, ou en combinaison, la dégradation de la vitamine A.

L'activité vitaminique $\mathrm{A}$ est liée à la présence simultanée du noyau $\beta$-ionone et de la chaîne latérale polyénique et du maintien de leurs doubles liaisons. La destruction sous l'influence de l'oxygène est due principalement à la saturation de ces doubles liaisons.

L'étude de l'influence de la lumière fluorescente sur le taux de vitamine A et de carotène du lait a montré (Sintra 1963) au cours des premières heures d'irradiation, une augmentation du taux des vitamines $\mathrm{A}$, imputable à la transformation du $\beta$-carotène.

Diverses substances, partiellement caractérisées, ont pu être mises en évidence (Sintra 1962) après photo-décomposition du $\beta$-carotène, par chromatographie sur couche mince.

Dans leur analyse du comportement de la vitamine A dans le lait, van der Mijll Dekker et Engel (1952) ne trouvent pas de perte de vitamine A due à la stérilisation seule; par contre, le stockage de ce lait stérilisé à la température ordinaire et à la lumière entraîne un abaissement de 30 p. 100 de l'activité vitaminique A en 6 mois ; il est de 11,7 p. 100 déjà après 10 jours. Selon Wagner (1953) la croissance des rats nourris au lait stérilisé ne redevient normale qu'après administration de vitamine $A$.

Pour Herreid (1956), la stérilisation conduit à une perte de 10 p. 100 de la teneur initiale en vitamine A.

Les valeurs trouvées par Wodsack (1960) sont les plus défavorables, mais vraisemblablement les plus valables. Elles montrent que la stérilisation (60 minutes à $115^{\circ} \mathrm{C}$ ) conduit à une perte de 
30-35 p. 100 de la vitamine A, alors que l'autoclavage produit une perte de 25 p. 100 et la cuisson 5 minutes à ébullition sous agitation à l'air, $18-22$ p. 100 .

Les traitements nécessaires à la préparation du lait condensé maintiennent 75-78 p. 100 du contenu initial du facteur A.

Après pasteurisation (10-20 minutes à $\left.71-74^{\circ} \mathrm{C}\right)$ le lait contient encore 84-90 p. 100 de la teneur initiale.

Si l'on se base sur le travail de Herreid (1956), le traitement à ultra-haute température $\left(138-149^{\circ} \mathrm{C}\right)$ pendant un laps de temps de l'ordre de la seconde - genre de traitement qui comprend l'upérisation et que nous désignerons désormais par U. H. T. maintient plus des 90 p. 100 de l'activité vitaminique A initiale, ceci même après trois mois de stockage à $4^{\circ} \mathrm{C}, 21^{\circ} \mathrm{C}$ ou $37^{\circ} \mathrm{C}$.

Signalons encore que les métaux, tout particulièrement le cuivre, le cobalt et le manganèse agissent comme catalyseurs du transport d'oxygène et de la destruction de la vitamine A.

\section{Vitamine}

La teneur initiale très variable du lait en vitamine $\mathrm{D}$ est sous l'influence des conditions d'éclairage et d'alimentation du bétail. Ainsi, le lait d'été peut contenir 4 fois plus de vitamine D que celui d'hiver.

Selon S. Fuchs (1953) qui signale les données de Bernhard, le lait upérisé présenterait la même teneur en vitamine D que le lait pasteurisé ; le lait autoclavé serait encore moins atteint. La vitamine D est relativement très stable à la chaleur en présence ou absence d'air. L'actinisation ou l'irradiation de la lumière normale n'a que peu d'effet sur la vitamine D que ce soit par élévation de l'activité vitaminique par transformation de stérols inactifs en composés actifs (on ne connaît pas bien la teneur en provitamine D du lait) ou que ce soit par transformation de la vitamine D par oxydation. Des produits d'oxydation, même s'ils apparaissent, présentent souvent une activité antirachitique : la cétone 250 par exemple. (Raoult et coll. 1956).

L'hydrogénation totale de la vitamine $\mathrm{D}$ conduit à des composés ayant perdu toute activité antirachitique mais qui manifestent par contre une activité hypercalcémiante. Cependant, les manipulations habituelles du lait ne comportent pas de traitements réducteurs suffisant à atteindre ce stade.

Nous laisserons de côté les vitamines $\mathrm{E}$ et $\mathrm{K}$, assez stables, mais dont l'importance dans le lait et les produits dérivés est limitée par une faible concentration naturelle, insuffisante à elle seule à couvrir les besoins.

\section{Vitamine $B_{1}$}

Dans le lait, l'activité vitaminique $B_{1}$ est à répartir entre la thiamine et le pyrophosphate de thiamine, l'une et l'autre pouvant 
se trouver soit à l'état libre, soit à l'état lié de manière plus ou moins lâche aux protéines lactosériques. La forme liée est prédominantes dans le colostrum. (Kon 1951). L'oxydation de la thiamine donne lieu à la formation du thiochrome par déhydrogénation, élimination d'eau et formation d'un noyau intermédiaire entre les structures pyrimidique et thiazolique initiales. Le thiochrome bleu ne possède plus aucune activité vitaminique.

Les diverses influences de stockage et des traitements industriels sur la teneur en thiamine totale des laits sont reportées sur la figure 1 .

Pendant les premiers jours de stockage du lait cru ou pasteurisé, une augmentation du taux initial de thiamine peut intervenir à la suite de la biosynthèse par les bactéries lactiques présentes ou qui se sont maintenues (thermophiles) dans le lait, (Davidov et Goulko, 1964).

La quantité de $\mathbf{B}_{1}$ fournie au nourrisson par le lait de vache est suboptimale, une diminution de sa teneur de l'ordre de $\mathbf{7 5}$ p. 100 environ, comme c'est le cas dans le lait autoclavé (Holt et coll. 1949) conduit, dans l'urine du jeune enfant, à une diminution anormale (moins de $4 \mathrm{~g} / \mathrm{j}$.) en dessous de la limite admise de l'équilibre physiologique comme témoin.

\section{Vitamine $\mathbf{B}_{2}$}

Le lait constitue pour l'homme une source très importante de vitamine $B_{2}$. Les $9 / 10$ sont sous forme libre, tandis que 10 p. 100 sont combinés aux protéines, sous forme de flavine-adéninedinucléotide, et peuvent être enzymatiquement reconvertis en forme libre dans le lait lui-même.

Alors que le stockage à l'obscurité et les traitements thermiques ne l'affectent guère, la vitamine $B_{2}$ est très photosensible comme le montrent les études de Kon (1951), van der Mijll Dekker et Engel (1952), Stamberg et Theophilus (1944) et Randoin et Causeret (1952). Ces derniers ont pu mesurer un abaissement de 85 p. 100 du taux initial en une heure d'exposition au soleil. (Voir fig. 2).

De l'étude de l'action de divers types de lumière artificielle sur le taux en vitamine $B_{2}$ et $C$ du lait en bouteilles blanches ou brunes ou encore de berlingots (Somogyi et Ott, 1962), il ressort que les tubes lumineux jaunes type 16 sont les seuls qui n'entraînent pas de perte trop importante des 2 vitamines considérées. Ceci est à mettre en relation avec le fait que les tubes jaunes ne présentent qu'une à deux lignes spectrales dans le domaine de 550 à $600 \mu$, soit dans la zone limite du spectre critique pour l'influence sur les vitamines $\mathbf{B}_{2}$ et $\mathbf{C}$.

En ce qui concerne la vitamine $B_{2}$ seule, les tubes lumineux blancs chauds de luxe (type 32 ) donneraient des résultats relativement proches de ceux obtenus avec les tubes jaunes. Nous reviendrons sur le sujet de la destrution de la vitamine $B_{2}$ par la lumière dans le cadre plus détaillé de l'étude de l'oxydation. 


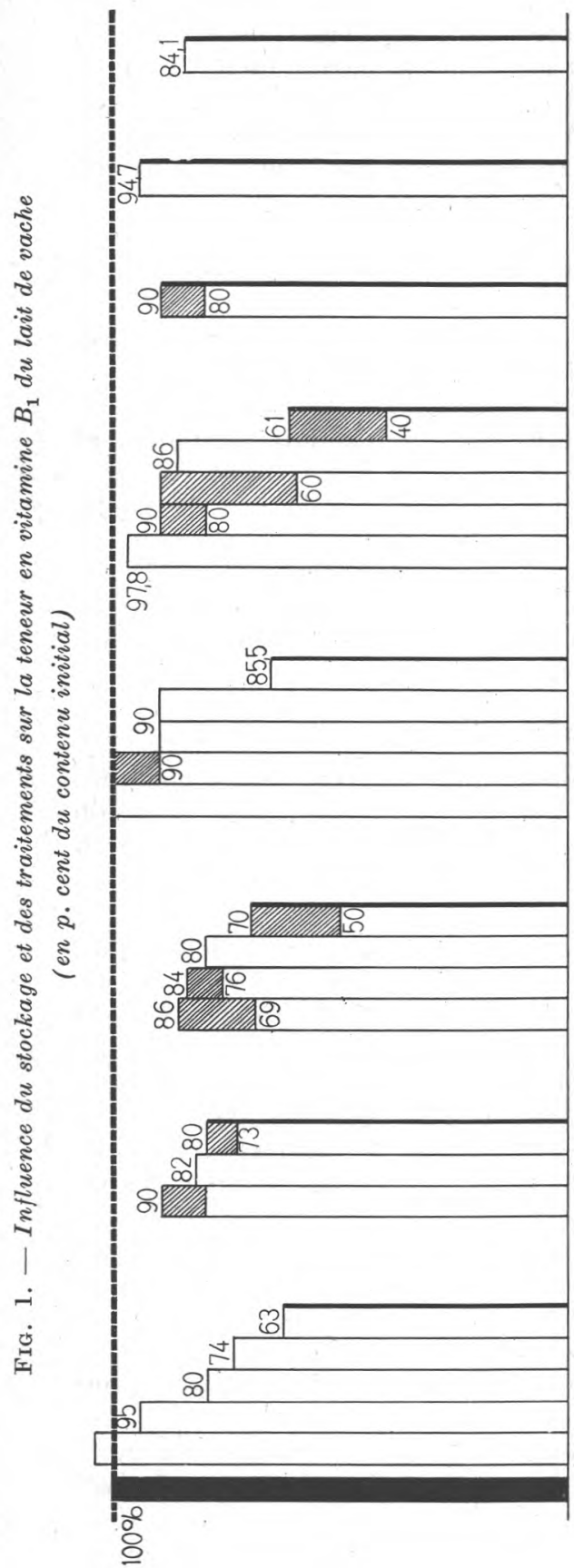

Boisselot et Causeret

명 Davidov et Goulko

Davidov et Goulko

Kon

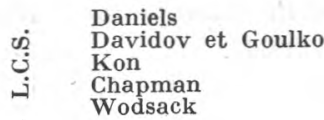

Bernhard

Fuchs

Herreid

Chapman

Gregory et Burton

Hostettler

Henry et Kon

Gregory et coll.

Hostettler

Wodsack

Dekker et Engel

Wodsack

Fuchs

Kendall

Hadj Hambi

Hostettler

Dekker et Engel

Davidov et Goulko
1946

1954

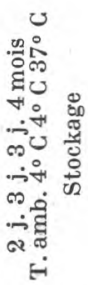




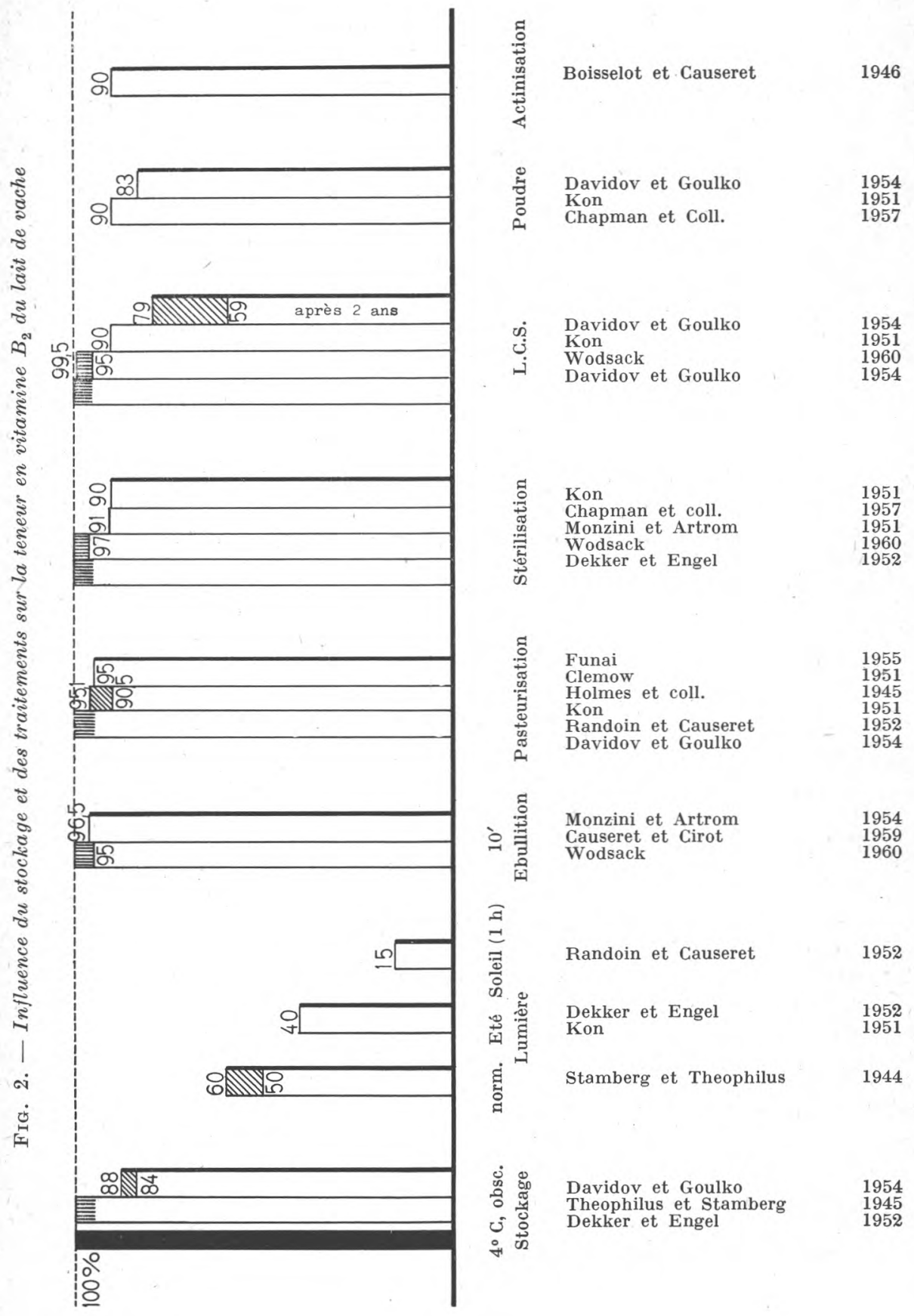




\section{Acide pantothénique}

L'acide pantothénique est répandu dans la nature et le lait en contient sous forme libre un taux suffisant pour que 2,9 litres satisfassent le besoin quotidien. De l'avis unanime des expérimentateurs, les traitements du lait et son stockage n'ont que peu d'influence sur la teneur en acide pantothénique. Alors que le stockage, l'exposition à l'air, la préparation du lait condensé et en poudre n'atteignent aucunement le taux initial d'acide pantothénique, la pasteurisation entraînerait une perte de 10 p. 100. (Chapman et coll. 1957, Kon et Thompson, 1957).

\section{Biotine}

La biotine dont un litre de lait de vache contient 1/10 du besoin journalier, est assez stable et son taux initial se maintient au cours des traitements tels le stockage à la lumière, la congélation (Lawrence et coll. 1946), la pasteurisation (Randoin et Causeret, 1952), la préparation du lait condensé et du lait en poudre (Hodson, 1945). Les plus grandes pertes signalées, (Kon et Thompson, 1957 ; Chapman et coll. 1957) atteignent 10 p. 100 pour la pasteurisation et la stérilisation, $10-15$ p. 100 pour le séchage et la condensation.

\section{Vitamine $\mathbf{B}_{6}$}

Les trois formes de la vitamine $B_{6}$, pyridoxine, pyridoxal et pyridoxamine, sont présentes dans le lait. Toutefois, c'est la forme aldéhyde qui prédomine. Le pyridoxal représente en effet $75-80$ p. 100 de vitamine $B_{6}$ lactique (Rabinowitz et Snell, 1948) ; (Gregory, 1959), alors que 21 p. 100 environ se trouvent sous forme de pyridoxamine et $4 \mathrm{p} .100$ sous forme de pyridoxine.

Or, il se trouve que, selon György, (1954) e'est précisément la forme manquante qui est la plus résistante à la chaleur. C'est elle également qu'il est recommandé d'ajouter au lait pour effectuer une supplémentation adéquate. Le taux est à contrôler de très près dans les préparations commerciales pour éviter la répétition de ce drame terrible qui, voici quelques années, a atteint de convulsions pseudo-épileptiques des nourrissons recevant un lait industriel qui ne contenait plus que des traces de vitamine $B_{6}$.

La figure 3 présente l'influence des divers traitements sur le taux en vitamine $B_{6}$ du lait.

Dans la préparation du lait évaporé par exemple, il y a perte énorme de pyridoxal (45-60 p. 100) mais une légère élévation de pyridoxamine (Gregory, 1959; Hodson, 1956). Le stockage subséquent à la température ordinaire du lait évaporé ou stérilisé, entraîne une perte supplémentaire (30 p. 100) de l'activité vitaminique $B_{6}$. La plus grande partie de l'activité $B_{6}$ résiduelle n'est alors liée ni au pyridoxal ni à la pyridoxamine mais à la pyridoxine. 


\section{Vitamine $\mathbf{B}_{12}$}

Les deux formes hydro- et cyano-cobalamine de cette vitamine se trouvent dans la proportion de 84 et 16 p. 100 respectivement (Sreenivasamurthy et coll., 1953) dans le lait, soit sous forme combinée aux protéines, soit sous forme libre. Le stockage n'entraîne pas de perte des $0,01 \mathrm{mg} / \mathrm{l}$ que contient le lait. La pasteurisation n'affecte que peu le taux de $B_{12}$ tandis que les autres traitements : stérilisation, ébullition, préparation de poudre de lait et de lait condensé, infligent des pertes sérieuses, comme on peut le voir sur la figure 4 .

\section{Vitamine $\mathbf{C}$}

Il faut remarquer d'emblée que plus que pour toute autre vitamine, les pertes en acide ascorbique (et subsidiairement en vitamine A) du lait sont très fortement accrues par le stockage et le vieillissement préalable du lait. Pendant cette période, c'est la lumière qui amorce la dégradation de l'acide ascorbique, bien que celui-ci, sans couleur, ne puisse l'absorber lui-même. Il sera oxydé par une réaction photochimique dans laquelle la vitamine $B_{2}$ joue le rôle d'un agent sensibilisateur.

L'oxygène est nécessaire pour le déroulement de ce processus qui se produit en deux étapes :

a) Transformation réversible de l'acide ascorbique en acide déhydroascorbique, résistant à la chaleur et présentant encore une activité vitaminique.

b) Décomposition plus poussée à partir de l'acide déhydroascorbique, même à l'abri de la lumière et dans laquelle intervient le cuivre et la vitamine $\mathrm{B}_{2}$.

La chaleur augmente alors cette vitesse d'oxydation de manière très sensible. Dunkley et coll. (1962) analysent les facteurs qui font apparaître des goûts étrangers dans le lait dans des conditions d'exposition à la lumière qui ne font pas intervenir l'oxydation des graisses. Ils distinguent le goût de lumière et le goût d'oxydation dû spécifiquement à l'influence de la vitamine C et montrent quelle est la susceptibilité du lait à former l'un et l'autre après homogénéisation, adjonction de cuivre et traitement à l'ascorbate-oxydase. Voici les résultats :

\begin{tabular}{|c|c|c|}
\hline & \multicolumn{2}{|c|}{ Susceptibilité au } \\
\hline & Goût de lumière & Goût d'oxydation \\
\hline $\begin{array}{l}\text { Homogénéisation } \ldots \ldots \ldots \ldots \ldots \ldots \\
\text { Présence de cuivre } \ldots \ldots \ldots \ldots \ldots \\
\text { Ascorbate oxydase } \ldots \ldots \ldots \ldots \ldots \ldots \\
\text { Ascorbate ajouté. } \ldots \ldots \ldots \ldots \ldots \ldots\end{array}$ & $\begin{array}{l}\text { accrue } \\
\text { accrue } \\
\text { abaissée } \\
\text { nulle }\end{array}$ & $\begin{array}{l}\text { abaissée } \\
\text { accrue } \\
\text { nulle } \\
\text { abaissée }\end{array}$ \\
\hline
\end{tabular}




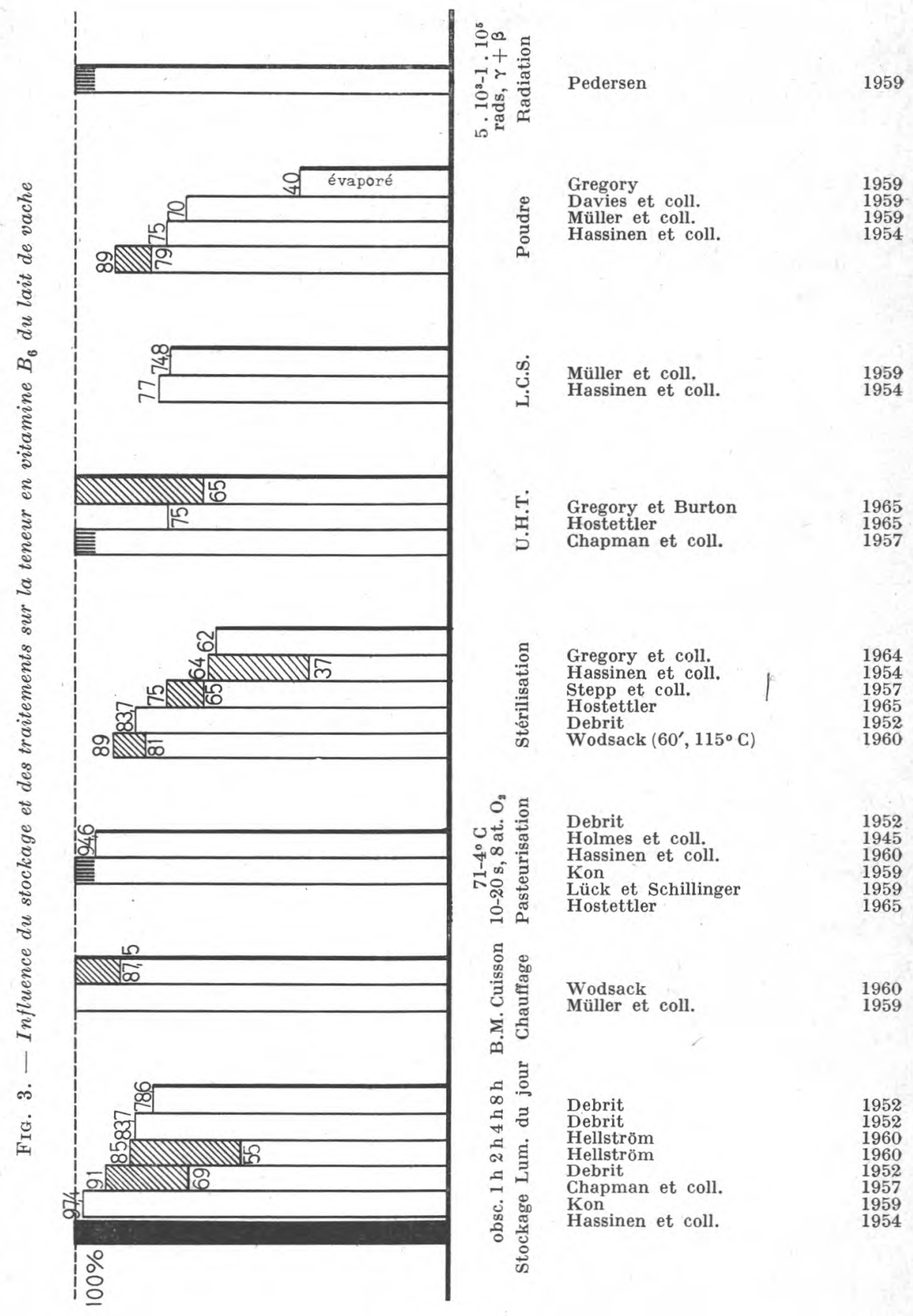




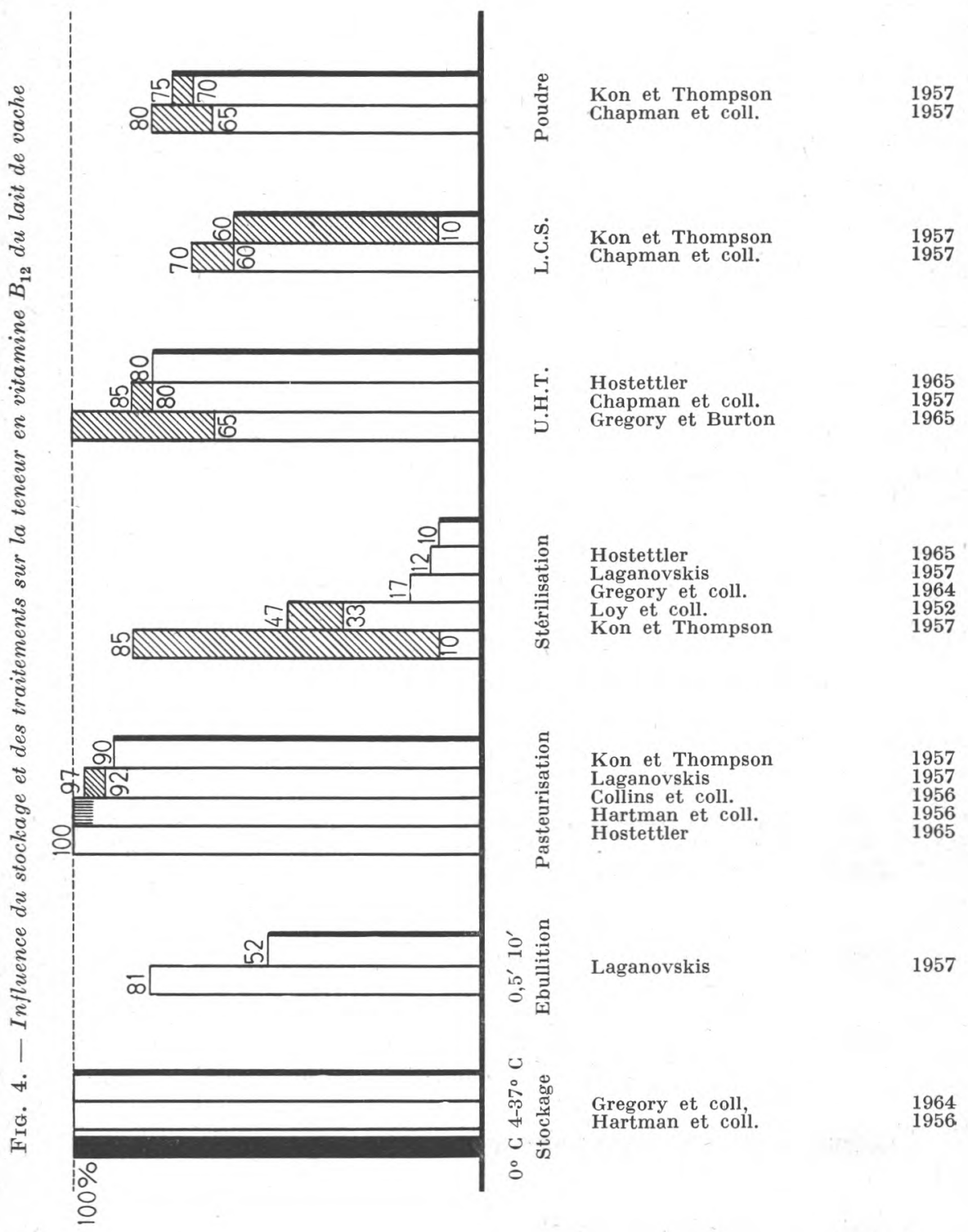


FIG. 5. - Influence du stockage et des traitements sur la teneur en vitamine $C$ du lait de vache

cru

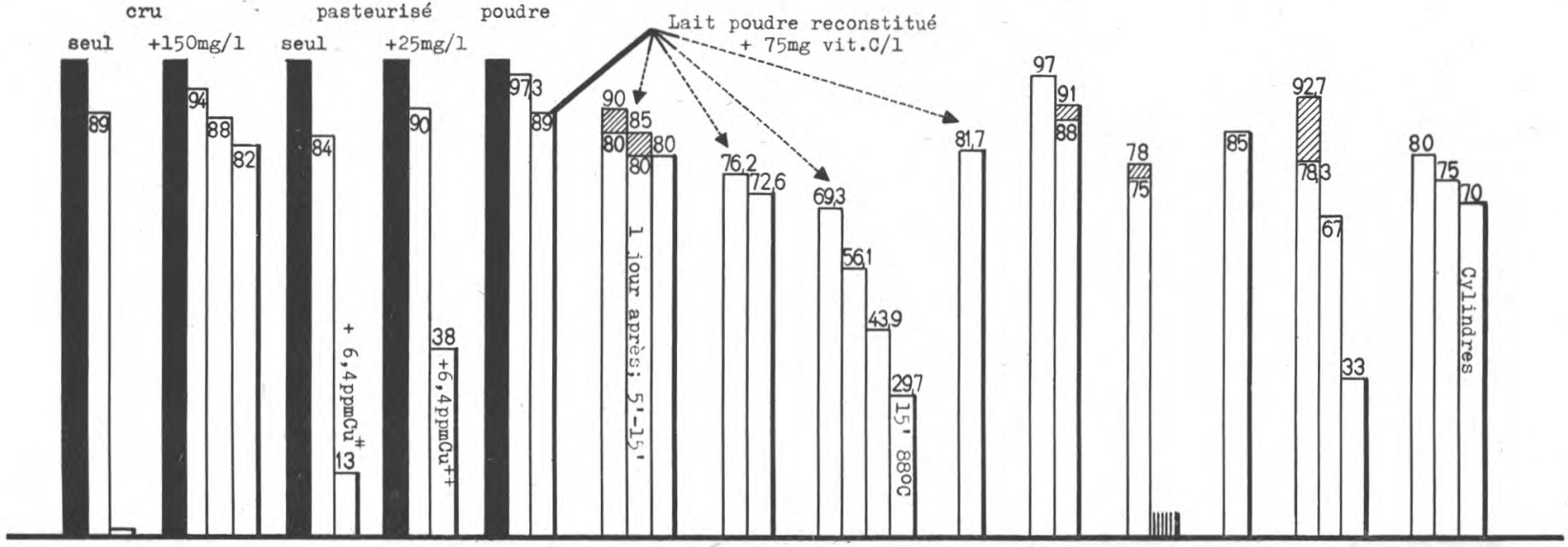

$\underset{4^{\circ} \mathrm{C}}{3 \text { j. } 2 \text { m. } 1 \text { j. } \underset{4^{\circ}}{2} \text { j. } 3 \text { j. }} \quad 2 \underset{4^{\circ} \mathrm{C}}{\text { Stockage }}$

1 an

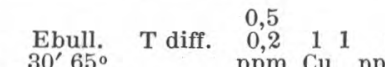

$30^{\prime} 65^{\circ}$ ppm Cu ppm Fe

Chaleur Lumière Cuivre Fer Pasteur. Steril. U.H.T. L.C.S. Poudre

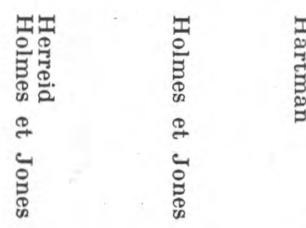

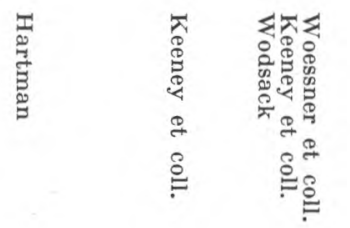

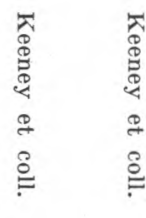

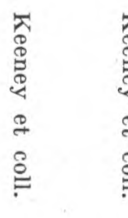

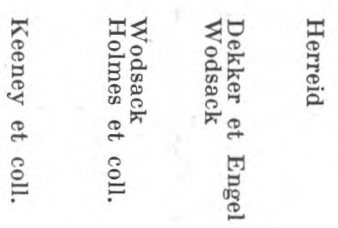

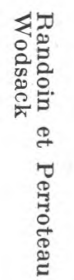

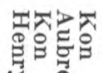

(9)

$\stackrel{8}{\varrho}$ 
Les effets délétères s'additionnent :

Lumière $+\mathrm{O}_{2}+$ Homogénéisation $+\mathrm{Cu}+$ Chaleur + influence de la vitamine $\mathrm{B}_{2}$ (voir sous photo-oxydation).

Une très intéressante revue des problèmes des goûts étrangers et de leur apparition dans le lait de consommation a été présentée par Ritter (1962) au 16 ${ }^{\mathrm{e}}$ Congrès International de Laiterie.

Il semble que la destruction dépend plus du temps pendant lequel l'on maintient la température que de la température ellemême. Ainsi, si l'on admet un effet de pasteurisation de 10 p. 100 environ, l'upérisation abaisserait de 22,8 p. 100 le taux de vitamine C, soit jusqu'à 77,2 p. 100 et la stérilisation de 45,9 p. 100 amenant le taux résiduel à 54,1 p. 100 du contenu initial (Fuchs 1953).

Les données reportées sur la figure 5 permettent de juger de l'importance relative des divers traitements sur le taux en vitamine C du lait.

\section{Photo-oxydation}

D'une manière générale, les nombreuses études effectuées sur les effets de la lumière sur l'oxydation et l'apparition des goûts du lait ont montré :

1) que les goûts d'oxydation et de lumière sont induits par la lumière ;

2) que l'absence d'air et particulièrement d'oxygène en retarde l'apparition ;

3) que les longueurs d'ondes inductrices de ces goûts se situent dans le domaine du spectre visible.

4) enfin que la riboflavine est directement impliquée dans leur formation.

L'examen du spectre d'absorption de la vitamine $B_{2}$ et sa comparaison avec le spectre de l'énergie solaire en fonction des longueurs d'onde, montre que la vitamine $\mathrm{B}_{2}$ absorbe dans une partie riche en énergie du spectre solaire. Il y a activation de la vitamine $\mathrm{B}_{2}$ capable de produire par la suite, à son tour, des réactions photochimiques.

Une étape dans l'explication du mécanisme de cette action a été franchie récemment par Singleton et coll. (1963) et Aurand et coll. (1964).

Ces auteurs ont tout d'abord confirmé que le goût ne peut être induit dans le lait écrémé dialysé sans adjonction de vitamine $\mathrm{B}_{2}$.

S'appuyant sur des observations d'Isenberg et Szent-György (1958) relatives au passage de la riboflavine sous forme semiquinoïde par transfert, en milieu neutre, d'électrons du tryptophane, de protéine contenant du tryptophane ou de molécules structurellement apparentées au tryptophane, ils ont ensuite montré qu'une relation étroite existe entre : 
- la destruction à $450 \mathrm{~nm}$ de la riboflavine d'une part, et

- l'apparition du goût d'oxydation, d'autre part.

L'examen des spectres d'absorption indique que les protéines du lait fixent la riboflavine en formant avec elle un complexe labile par l'intermédiaire du tryptophane.

Ce complexe protéine-tryptophane-riboflavine absorbe l'oxygène et, sous l'influence de l'énergie des photons, est le siège d'un transfert d'électrons qui, après un stade transitoire, excité, aboutit à la formation de deux produits nouveaux :

1) forme oxydée de la protéine et

2) produit de décomposition de la riboflavine par photolyse.

De plus, la vitamine $B_{2}$ activée par l'énergie des photons, atteint la vitamine $\mathrm{C}$ qui elle, prise isolément, n'est pas affectée par la lumière visible. A la faveur d'une réaction photo-chimique dans laquelle la vitamine $B_{2}$ joue le rôle d'agent sensibilisant, la vitamine $\mathrm{C}$ est oxydée en acide déhydroascorbique, dégradé à son tour, même à l'abri de la lumière, en diverses substances (voir aussi page 153).

Ces substances provoquent par elles-mêmes ou après réaction avec d'autres composés du lait, des goûts de lumière et d'oxydation ; les acides oxalique et thréonique seraient le résultat de ces dégradations en chaîne.

Une oxydation plus poussée de la vitamine $B_{2}$ détruit son activité vitaminique et la transforme en lumichrome si le milieu est neutre ou acide et en lumiflavine en milieu alcalin. Une photolyse subséquente en aérobiose, activée par la présence de fer, scinde ces produits en composés tels l'acide formique et l'acide glycolique.

En résumé, la forme activée de la vitamine $B_{2}$ interviendrait sur les protéines par l'intermédiaire du tryptophane qu'elles contiennent, sur la prise d'oxygène par le complexe protéine-tryptophaneriboflavine et sur la décomposition de la vitamine $\mathrm{C}$ et la formation subséquente des goûts de lumière et d'oxydation.

Récemment Abbott et Waite (1965) ont poursuivi l'étude de l'influence des substances anti-oxydantes pour le maintien de la qualité du lait. Les agents choisis ont été principalement le tocophérol et le dodécyl-gallate.

L'expérience a montré que le taux des peroxydes (mEq/kg mat. grasse) de 4,0 après $160 \mathrm{j}$ à $37^{\circ} \mathrm{C}$ dans la poudre de lait de contrôle, n'est plus que 3,1 après traitement à 0,01 p. 100 de vit. $\mathrm{E}$ et de 0,5 seulement après introduction de 0,01 p. 100 de dodécylgallate.

La mesure de la consommation d'oxygène corrobore ce résultat : $19,5 \mathrm{mg} / 100 \mathrm{~g}$ de poudre dans le contrôle, $17,0 \mathrm{mg} / 100 \mathrm{~g}$ dans la poudre traitée au tocophérol et $9,3 \mathrm{mg} / 100 \mathrm{~g}$ dans celle ayant reçu le dodécyl-gallate. 
Il est possible d'imaginer la protection des tocophérols à leur tour par l'acide ascorbique ainsi que le travail de Krukovsky (1964) le suggère et de réaliser ainsi, au moyen de vitamines, une synergie protectrice telle celle bien connue de l'expérience de Privett et Quackenbusch (1954). Cette dernière montre l'abaissement de l'indice de peroxydes formés dans une graisse animale sous l'influence de traitements au tocophérol et à l'ensemble : tocophérol + acide ascorbique.

\section{Conclusions}

La question de la protection chimique contre la photo-oxydation nous introduit déjà dans la considération des mesures de prévention de l'oxydation et de la destruction vitaminique à instaurer ou à améliorer. Ces mesures, relativement simples, reposent sur les principes généraux suivants :

1) abaissement du délai d'attente entre la production du lait, sa réfrigération et son utilisation. Il est souhaitable d'envisager une extension à tous les laits de consommation d'une châ̂ne $d u$ froid continu. Celle-ci peut se composer, par exemple, d'une réfrigération immédiate (tank réfrigéré) au local de ramassage, d'un transport en camion avec citerne isolée thermiquement, suivis d'un stockage et d'un conditionnement à basse température dans la centrale régionale.

La température du lait ne serait élevée que pour les phases d'homogénéisation, et de pasteurisation.

2) Protection systématique contre la lumière et instauration de ce que l'on pourrait appeler " une chaîne noire " continue de la ferme au consommateur. Ce principe de la chaîne noire, complément logique à la chaîne du froid, n'est cependant pas suffisamment pris en considération actuellement.

3) Elimination de l'oxygène dissous dans le lait lors de son utilisation dans les processus industriels. Le dégazage et le remplacement éventuel par des gaz inertes (pour les poudres de lait spécialement) permet d'éliminer pratiquement complètement l'oxygène du lait ou des produits dérivés. Pour diminuer les risques d'oxydation il faut veiller, à chaque étape de transformation, à éviter les contacts avec tous les agents oxydants.

4) Amélioration des conditions du traitement thermique, par variation des facteurs du produit : $\mathrm{T} \times \mathrm{t}$. $(\mathrm{T}=$ Température $; \mathrm{t}=$ temps de traitement à haute température). Les développements récents des procédés U. H. T. (ultra-haute-température) démontrent l'importance de l'obtention d'un équilibre optimum entre le temps et la température du traitement pour aboutir à une stérilisation maximale tout en prévenant des dommages importants au lait.

Le même principe est applicable à la détermination des meilleures conditions de pasteurisation 
5) Photo-protection par synergie vitaminique. Il est possible d'envisager d'ores et déjà un abaissement du produit $\mathrm{T} \times \mathrm{t}$, en maintenant une pasteurisation ou une stérilisation efficaces par l'utilisation d'antibiotiques spéciaux "naturels" et dégradés dans le tube digestif. La nisine en est un qui permet d'inhiber le développement bactérien (de nombreux germes, dont le Colostridium par exemple) et qui est rapidement détruit par les enzymes intestinaux. Il apparaît naturellement dans certains fromages. Il est dès lors indiqué de l'utiliser, après isolement, comme additif bactériostatique alimentaire (Hawley 1958; Gregory, Henry et Kon, 1963).

Ce programme est constamment en voie de développement et d'exécution; il y a de bons espoirs d'aboutir, dans un délai relativement rapproché, au maintien des vitamines naturelles du lait ou de celles qui lui ont été ajoutées, afin de lui permettre de soutenir sa réputation d'aliment le plus complet de la nutrition humaine.

\section{Summary}

Milk can be considered as an important source of vitamins for newborns, children and adults. In light of recent findings, it has been shown that a normal daily consumption can cover the total needs in vitamin $B_{1}, B_{2}, B_{6}, B_{12}$, pantothenic acid, inositol, and can provide a large part of needs for vitamins $A, D$, biotin and ascorbic acid.

The facts concerning the stability of each of these vitamins under the influence of various thermal treatments and of light radiation which is substantial during storage, have been assembled. The tables resume the values found by numerous authors.

Photo-oxidation and the causes of the appearance of foreign tastes have been discussed. Various methods for avoiding photooxidation and vitamin depletion have been envisaged. They involve a cold chain, a "black chain ", elimination of oxygen disolved in the milk, improvement in the thermal treatment utilizing "biodegradable " antibiotics, photo-protection by vitamin synergism.

\section{RÉFÉRENCES BIBLIOGRAPHIQUES}

Аввот J. et Watte R. - J. Dairy Res, 1965, 32, 143.

Aurand L. W., Singleton J. A. et Matrone G. - J. Dairy Sci, 1964, 47, 827.

Berhnard K., Gschaedler L. et Sarasin A. - Schweiz. Akad. Med. Wissenchaften 1953, 9, 5-6, 312 .

Boisselot J. et Causeret J. - Bull. Soc. Sci. Hyg. Alim. 1946, 34, 176.

Causeret J. et Cirot E. - Lait 1959, 39, 159.

Chapman H. R., Ford J. E., Kon S. K., Thompson S. Y. et Rowland S. J. J. Dairy Res. 1957, 24, 2, 191.

Clemow N. J. - The New Zealand, J. Sci. Technol. A. Agric. Res. Sect. 1951, $32,6,14$. 
Collin R. - Thèse Doct. Méd. Vétér., Alfort (France) 1955.

Daniels A. L. - American J. Diseas. Children 1941, 62, 1, 127.

Davidor R. B. et Goulko L. - Inst. Biokhim im A. N. Bakha Sbornik, $1954,2,103$.

Davies M. K. - J. Dairy Res. 1959, 26, 2, 215.

Dekker van der Mijll L. P. et ENGel C. - Netherlands. Milk and Dairy J. $1952,6,104$.

Dietary Allowances. - Nat. Acad. of Sci. Nat. Res. Council Publication 1146, U.S.A., 1964.

DEBRIT F. P. - Intern. Zeisch Vitaminfors. 1952, 24, 331.

DRUmmond J. C. et Coward K. H. - Biochem. J. 1920, 14, 6, 734.

Drummond J. C. et Hilditch. - Empire Marketing Board rep. 1930, 35.

EDdy W. H. et KонмANN E. F. - Ind. and Engen Chem. 1925, 17, 69. 1931, $23,7,808$.

FunaI Y. - Tokushima. J. Exp. Med. 1955, 2, 3.

Fuchs A. - 13 e Congrès Int. de Lait, 1953, 3, 1018.

Gregory M. E. - J. Dairy Res. 1959, 26, 2, 203.

Gregory M. E., Henry K. M. et Kon S. K. - J. Dairy Res. 1964, 31, 113.

GyöRGY J. - J. Clin. Nutr. 1954, 2, 44.

Hамві Hadj. - Thèse Doct. Méd. Paris, 1956, 8, 45.

Hassinnen J. B., Durbin G. T. et Bernhart F. W. - J. Nutr. 1954, 53 , $2,249$.

Hartman A. M., Dryden L. P. et Riedel G. H. - J. Nutr. 1956, 59, 1, 77-102.

Hartman G. H. - A Study of Certain Oxidative Reactions in Milk, Thesis Rutgers University 1942 .

HAWLEY H. B. - Milchwissenschaft 1958, 13, 253.

Hellströ̀ V. - Int. Z. Vitaminforsch 1960, 30, 323.

Henry K. M. et Kon S. K. - J. Dairy Res. 1938, 9, 22-29.

Henry K. M., Houston J., Kon S. K. et Osborne L. W. - J. Dairy Res, $1939,10,272$.

Herreid E. O. - Le Lait, 1956, 36, 14-26.

Hodson A. Z. - J. Nutr. 1945, 29, 137.

Holmes H. N., Corbett R. E. et Hartzler. - In. Eng. Chem. 1936, 28, 133.

Holmes A. D., Lindeuist H. G., Jones C. P. et Wertz A. W. - J. Dairy Sci. 1945, 28, 29.

Holmes A. D. et Jones C. P. - J. Dairy Sci. 1948, 31, 99.

Holt L. E., Nemir R. L., Snyderman S. E., Albanese A. A., Keltron K. C. Guy L. P. et Carretero R. - J. Nutr. 1949, 37, 53.

Hopkins F. O. - Biochem. J. 1920, 14, 6, 725.

Hostettler H. - Travaux de Chimie Alimentaire et d'Hygiène 1965, 56, 137.

Isenberg I. et Szent-György A. - Proc. Acad. N.A.S. U.S. 1958, 44, 857.

Keeney D. G., BaUr M.S. L.S. et Garrett O. F. - Columbus, Ohio; The $J$. of Pediatr. $1949,36,745$.

Kendall N. - J. Pediatr. 1942, 20,65.

Kon S. K. - Ann. Nutr. Alim. 1951, 2, 211.

Kon S. K. - J. Dairy Research 1940, 11, 196.

Kon S. K. et Thompson S. Y. - Milchwissenschaft 1957, 12, 5, 166.

Krukovsky V. N. - New York State Coll. — J. Agric. Food Chem. U.S.A. $1964,12,3,289$.

Laganovskis-LatviJas S., Zinatwo P. S. R. - Akademias Vestis 1957,7, 81.

Lawrence J. M., Herrington B. L. et Maynard L. A. - J. Nutr. 1946, 32, 73.

Loy J. R. H. W., Haggerty J. F. et Kline O. L. - Ol. Ass, off. Agric. Chem. $1952,35,29$.

LÜ̈K H. et Sohillinger A. - Z. Lebensmitt. Untersuch, 1959, 110, 267.

Monzini A. et Artom A. - Ann. Sper. Agric. 1951, 5, 805, D.S.A., 14, 54.

Monzini A. et Artom A. - II. Latte, 1954, 28, 2, 77.

Müller U., Stenger K. et Wolf H. - Milchwissenschaft, 1959, 14, 115. 
Perdesen A. H. - Nord Mejeritidsskr 1959, 25, 40.

Privett O. S. et Guaekenbusch F. W. - J. Am. O.L. Chamb. Soc. 1954, $31,321$.

Rabinowitz J. C. - J. Biol. Chem. 1948, $176,1157$.

Randoin L. et Causeret J. - J. Alim. et Vie 1952, 40, 1, 2, 3, 223.

Raoult Y., Le Boulch N., Baron C., Bazier R. et Guerillot-Vinet A. Acad. Sci. 1956, 242, 3004.

Singleton J. A., Aurand L. W., Lancaster F. W. - J. Dairy Sci. 1963, $46,1050$.

Sremivasamurthy V., Nambudripad K. N., Iya K. K. - Indian. J. Diary Sci. $1953,6,3,105$.

Stamberg O. E. et Theophilus D. R. - Milk Dealer 1944, 33, 8, 32.

Stepp W., KÜHNAU J. et SoHRomder H. - Dans Die Vitamine und ihre klinische Anwendung. Vol. II, 7 ttr. édit. Stuttgart, 1957.

Somogyi J. C. et Отт E. - Für Vitaminforsch, 1962, 32, 4, 493.

Theophilus D. R. et Stamberg O. E. - J. Dairy Sci. 1945, 28, 4, 259.

WAGNER K. H. - Milchwissenschaft, 1953, 8, 364.

WoDsACK W. - Nahrung 1960, 4, 209.

Woessner W. W., Weckel K. G. et Schuette H. A. - J. Dairy Sci. 1940, 23, 1131 .

Zirva S. S. - Biochem. J. 1922, 16, 42. 